\title{
Diagnostic Accuracy of Combinations of Tumor Markers for Malignant Pleural Effusion: An Updated Meta-Analysis
}

\author{
Yuan Yang Ya-Lan Liu Huan-Zhong Shi \\ Department of Respiratory and Critical Care Medicine, Beijing Institute of Respiratory Medicine and \\ Beijing Chao-Yang Hospital, Capital Medical University, Beijing, China
}

\section{Keywords}

Tumor marker · Malignant pleural effusion · Diagnosis ·

Meta-analysis

\begin{abstract}
Background: The role of combinations of tumor markers such as carcinoembryonic antigen (CEA), carbohydrate antigens (CA) 125, 15-3, and 19-9, and CYFRA 21-1 (a fragment of cytokeratin 19) in diagnosing malignant pleural effusion (MPE) has not been clearly established. Objectives: This meta-analysis was performed to establish the overall diagnostic accuracies of combinations of these pleural fluid tumor markers for MPE. Methods: The PubMed, Ovid, Embase, Web of Science, and Cochrane bibliographic databases were searched. Sensitivity, specificity, and other measures of the accuracy of combinations of pleural CEA, CA 125, CA 15-3, CA 19-9, and CYFRA 21-1 in the diagnosis of MPE were pooled after a systematic review of English-language studies. $\boldsymbol{R} \boldsymbol{e}$ sults: Twenty studies met the inclusion criteria. For pleural fluid tumor marker combinations including more than 3 studies, the summary estimates of the sensitivity/specificity for diagnosing MPE were as follows: CEA + CA 125, 0.65/0.98; CEA + CA 15-3, 0.64/0.98; CEA + CA 19-9, 0.58/0.98; CEA + CYFRA 21-1, 0.82/0.92; and CA 15-3 + CYFRA 21-1, 0.88/0.94.
\end{abstract}

\section{KARGER}

(C) 2017 S. Karger AG, Basel

E-Mail karger@karger.com

www.karger.com/res
Conclusions: In patients with undiagnosed pleural effusion, the combinations of positive pleural CEA + CA 15-3 and CEA + CA 19-9 are highly suspicious for pleural malignancy, but the sensitivity of these tests is poor. Therefore, their routine role in the diagnostic algorithm of these patients is questionable, and management decisions should depend on positive cytological or biopsy results from the pleura.

(c) 2017 S. Karger AG, Basel

\section{Introduction}

Pleural effusions are common complications in patients with physical traumata or systemic disorders such as inflammation, infection, or cancer [1]. A malignant disease is one of the primary causes of pleural effusion. More than $90 \%$ of malignant effusions are due to metastasis, usually from primary tumors in the lung or breast [2]. The major diagnostic difficulty is differentiating between malignant and nonmalignant pleural effusion. Cytological analysis of pleural effusion is the most common method. However, the sensitivity of cytological analysis ranges from 40 to $70 \%$, as malignant cells may be absent, overlooked, or incorrectly identified from the samples [3-5]. 
Analysis of tumor markers in pleural effusions has been considered less invasive for differentiating malignant effusions from nonmalignant effusions. In 2008, we performed a meta-analysis reporting the overall diagnostic accuracy of pleural carcinoembryonic antigen (CEA), carbohydrate antigens (CA) 125, 15-3, and 19-9, and CYFRA 21-1 (a fragment of cytokeratin 19) for malignant pleural effusion (MPE). Our results showed that using 1 tumor marker alone for the diagnosis of MPE cannot be recommended, but the combination of 2 or more tumor markers seems to be more sensitive [6]. More recently, Nguyen et al. [7] performed a meta-analysis showing that the summary estimates of the sensitivity and specificity of pleural CEA, CA 15-3, CA 19-9, CA 125, and CYFRA 21-1 were as follows: CEA, 0.549 and 0.962; CA 15-3, 0.507 and 0.983; CA 19-9, 0.376 and 0.980; CA 125, 0.575 and 0.928; and CYFRA 21-1, 0.625 and 0.932. These figures were not as high as expected. On the other hand, the exact roles of combinations of these tumor markers remained controversial. Therefore, we performed a metaanalysis to update the overall diagnostic accuracies of combinations of pleural CEA, CA 125, CA 15-3, CA 19-9, and CYFRA 21-1 for MPE.

\section{Methods}

\section{Search Strategy}

Two authors independently searched Embase, Ovid, Web of Science, the Cochrane Database, and MEDLINE (using PubMed as the search engine) to identify relevant studies from January 1 , 2007, to October 30, 2015. The references before 2007 were based on the 2008 article by Liang et al. [6] and were included using the same inclusion standards and quality of methodology. The following search terms were used: "tumor marker," "combination," "carcinoembryonic antigen/CEA," "carbohydrate antigen 19-9/CA 19-9," "carbohydrate antigen 125/CA 125," "carbohydrate antigen 15-3/CA 15-3," "fragment of cytokeratin 19/CYFRA 21-1," "lung cancer," "malignant pleural mesothelioma," "pleural effusion/ pleural fluid," "sensitivity and specificity," and "accuracy."

\section{Inclusion Criteria}

Studies with a possible overlap of study populations (e.g., same authors, institutions, and period of study) were discussed by Y.-L.L. and Y.Y., and only the study with the best quality was used. The 2 reviewers independently judged study eligibility while screening the citations. Disagreements were resolved by consensus.

Studies were included in this meta-analysis if they met the following criteria: (1) the study addressed the potential of any combinations of the aforementioned markers in pleural fluid for diagnosing MPE; (2) all patients with pleural malignancies were established by the conventional "gold standard," i.e., MPE was confirmed through cytological study or pleural biopsy; and (3) the sensitivity and specificity of combinations of tumor markers were reported to provide sufficient information for constructing $2 \times 2$ contingency tables.

Combinations of Tumor Markers for

MPE

\section{Exclusion Criteria}

Studies were excluded from this meta-analysis for the following reasons: (1) they were not pertinent to any combination of the aforementioned markers; (2) MPE was not confirmed through cytological study or pleural biopsy specimens; (3) sensitivity and specificity were not given; (4) only a single tumor marker was examined; (5) only tumor markers in serum were examined; (6) the results were given in the form of a letter, abstract, review, case report, expert opinion, or nonclinical study; (7) they included fewer than 10 specimens; (8) they had duplicated or overlapping data; or (9) they were not based on human subjects.

\section{Data Extraction and Quality Assessment}

The final set of articles included was assessed independently by 2 reviewers (Y.-L.L. and Y.Y.). The 2 reviewers were blinded to publication details, and disagreements were resolved by consensus. The data retrieved from the reports included author(s), participant characteristics, publication year, test methods, sensitivity and specificity data, and methodological quality.

The methodological quality of the included studies was evaluated with the Quality Assessment of Diagnostic Accuracy Studies (QUADAS)-2 tool (online suppl. Appendix Fig. 3; for all online suppl. material, see www.karger.com/doi/10.1159/000468545) and the Standards for Reporting of Diagnostic Accuracy (STARD) score (max. score 25) $[8,9]$. In addition, for each study, the following study design characteristics were retrieved: (a) blinded (single or double) interpretation of determination and reference standard results; (b) consecutive or random sampling of patients; (c) crosssectional design (vs. case-control design); and (d) prospective data collection. We requested the relevant information from the authors unless the data on the above criteria were reported. If the authors did not respond, the "unknown" items were treated as "no."

\section{Statistical Analyses}

Standard methods recommended for meta-analyses of diagnostic test evaluations were used [10]. The data were compiled using Stata version 12.0 (Stata Corporation, College Station, TX, USA) and Meta-DiSc version 1.04 for Windows (Clinical Biostatics Unit, University Hospital Ramón y Cajal, Madrid, Spain). The following measures of test accuracy were computed: sensitivity, specificity, positive likelihood ratio (PLR), negative likelihood ratio (NLR), and diagnostic odds ratio (DOR). The $\chi^{2}$ and Fisher exact tests were used to detect statistical heterogeneity across the studies. A random-effects model was used to calculate the overall sensitivity, specificity, and other measures for each combination of pleural tumor markers $[11,12]$.

\section{Results}

\section{Study Characteristics and Quality of Reporting}

A total of 20 articles were included in the final analysis of the accuracies of combinations of pleural tumor markers in diagnosing MPE (Fig. 1) [13-32]. Eleven were from the 2008 article by Liang et al. [6]. Online supplementary Appendix Table 1 shows that in 9 studies (45.0\%) the

Respiration 2017;94:62-69

DOI: $10.1159 / 000468545$ 


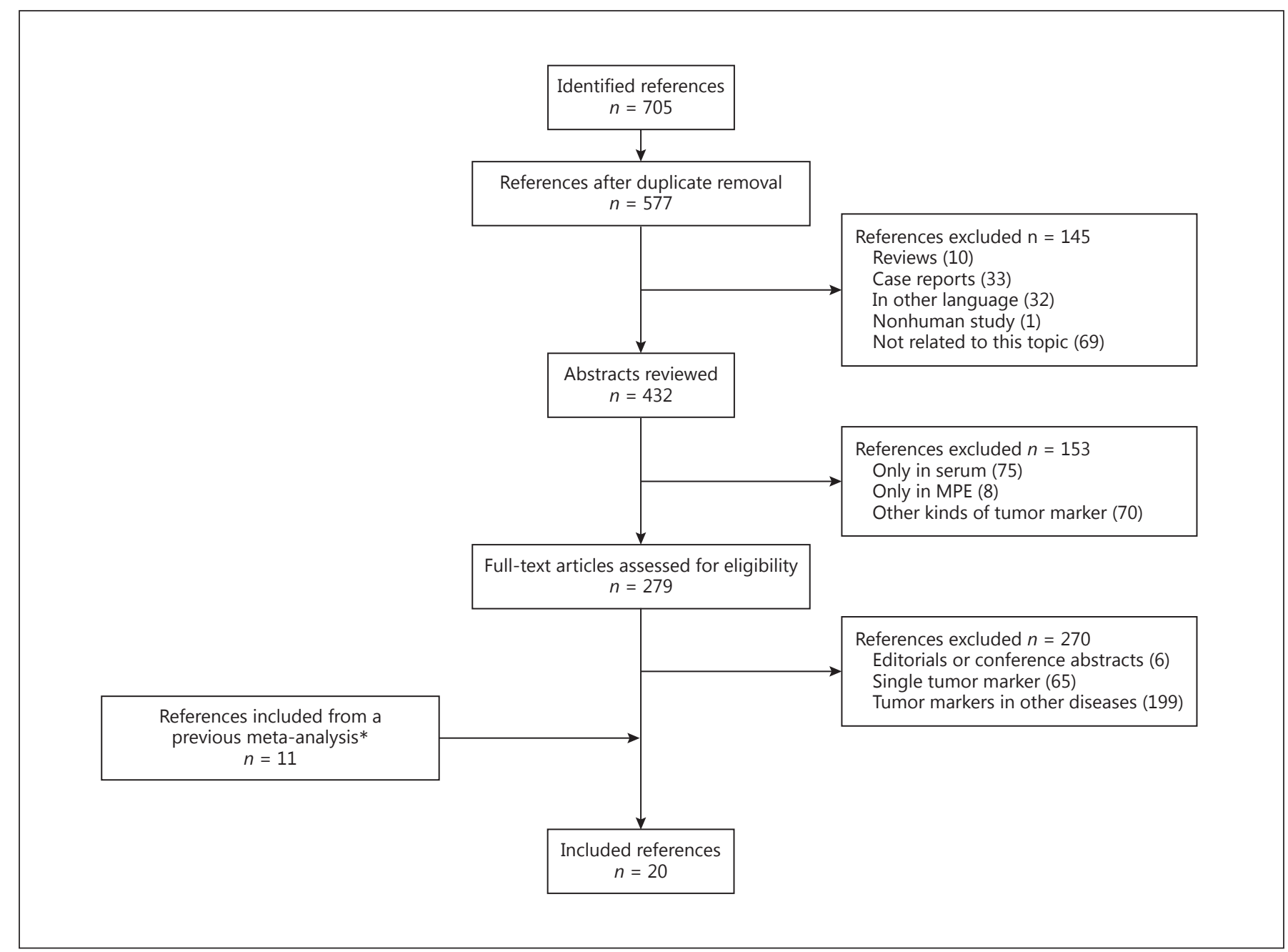

Fig. 1. Flow diagram of study selection and inclusion process. We searched 705 articles in the databases. A total of 426 records were removed after screening the titles and abstracts. Then, 270 references to editorial or conference abstracts, to a single tumor marker, and to tumor markers in other diseases were removed after

samples were collected from consecutive patients, that 6 studies $(30.0 \%)$ reported a blinded interpretation of tumor marker assays independent of the reference standard, and that 8 studies (40.0\%) had a prospective study design. The clinical characteristics, together with the STARD and QUADAS scores for each study, are outlined in online supplementary Appendix Table 2. In the present meta-analysis, all patients with pleural malignancies were established by the conventional "gold standard," i.e., MPE was confirmed through cytological study or pleural biopsy. screening the full texts. Eleven references before 2007 were included. * The references before 2007 are based on the 2008 article by Liang et al. [6]. In total, 20 articles were included in the current meta-analysis.

\section{Diagnostic Accuracy}

Figure 2 shows the forest plots of sensitivities and specificities for 5 kinds of combination of pleural tumor markers in diagnosing MPE. The corresponding summary receiver operating characteristic (SROC) curves are shown in online supplementary Appendix Figure 1.

The pooled sensitivities, specificities, PLR, NLR, and DOR for the pleural tumor marker combinations (Table 1) were: CEA + CA 125, 0.65, 0.98, 25.20, 0.346, and 66.08; CEA + CA 15-3, 0.64, 0.98, 28.81, 0.37, and 92.38; CEA + CA 19-9, 0.58, 0.98, 32.29, 0.43, and 112.22; CEA + CYFRA 21-1, 0.82, 0.92, 8.66, 0.176, and 68.73; and CA 


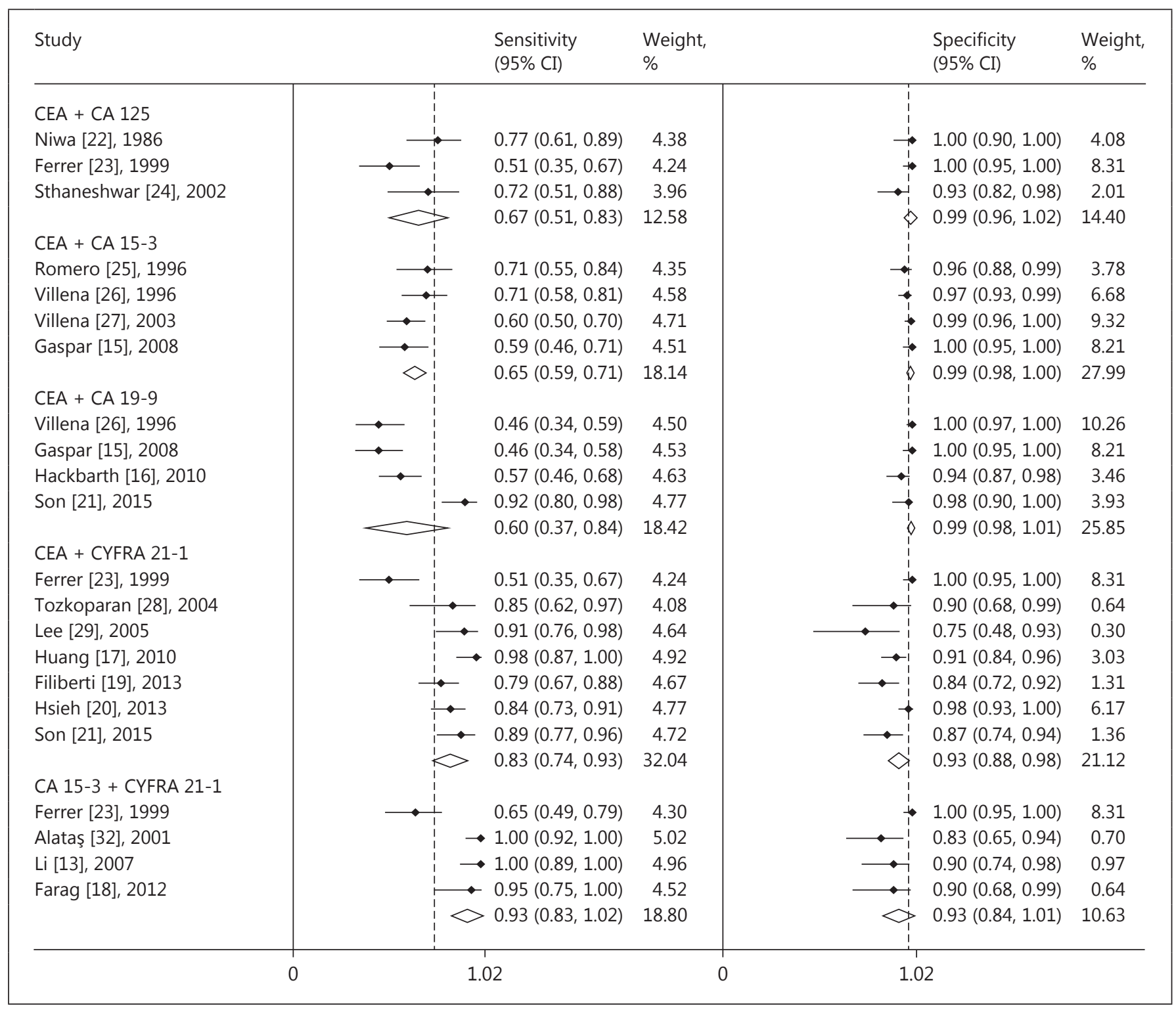

Fig. 2. Forest plots of estimates of sensitivity and specificity for assays of CEA + CA 125, CEA + CA 15-3, CEA + CA 19-9, CEA + CYFRA 21-1, and CA 15-3 + CYFRA 21-1 in the diagnosis of malignant pleural effusions. The point estimates of sensitivity and specificity from each study are shown as solid circles. The horizontal line crossing the solid circle represents the $95 \%$ confidence in-

15-3 + CYFRA 21-1, 0.89, 0.94, 8.83, 0.06, and 300.99, respectively. The pooled results of the diagnostic accuracy of other combinations that included less than 3 references are listed in online supplementary Appendix Table 3.

The $p$ values for sensitivity, specificity, PLR, NLR, and DOR for the combinations of tumor markers were not all

Combinations of Tumor Markers for

MPE terval (CI). The pooled sensitivities and specificities for the pleural tumor marker combinations were: CEA + CA 125, 0.67 and 0.99; CEA + CA 15-3, 0.65 and 0.99; CEA + CA 19-9, 0.60 and 0.99; CEA + CYFRA 21-1, 0.83 and 0.93; and CA 15-3 + CYFRA 21-1, 0.93 and 0.93 . CEA, carcinoembryonic antigen; CA, carbohydrate antigen.

above 0.05 (Table 1), indicating heterogeneity between the studies. For this reason, the random-effects model was determined to be the most appropriate pooled estimator for diagnostic accuracy.

The SROC curve and the area under the curve (AUC) present an overall summary of test performance and display the trade-off between sensitivity and specificity. As 
Table 1. Pooled results of diagnostic accuracy of CEA + CA 125, CEA + CA 15-3, CEA + CA 19-9, CEA + CYFRA 21-1, and CA 15-3 + CYFRA 21-1 in malignant pleural effusion

\begin{tabular}{|c|c|c|c|c|c|c|c|c|c|c|c|c|c|}
\hline $\begin{array}{l}\text { Combina- } \\
\text { tion }\end{array}$ & N1 & N2 & $\begin{array}{l}\text { Sensitivity } \\
(95 \% \mathrm{CI})\end{array}$ & $\begin{array}{l}\text { Hetero- } \\
\text { geneity }{ }^{\mathrm{a}} \\
(p)\end{array}$ & $\begin{array}{l}\text { Specificity } \\
(95 \% \mathrm{CI})\end{array}$ & $\begin{array}{l}\text { Hetero- } \\
\text { geneity } \\
(p)\end{array}$ & $\begin{array}{l}\text { PLR } \\
(95 \% \mathrm{CI})\end{array}$ & $\begin{array}{l}\text { Hetero- } \\
\text { geneity } \\
(p)\end{array}$ & $\begin{array}{l}\text { NLR } \\
(95 \% \text { CI })\end{array}$ & $\begin{array}{l}\text { Hetero- } \\
\text { geneity } \\
(p)\end{array}$ & $\begin{array}{l}\text { DOR } \\
(95 \% \mathrm{CI})\end{array}$ & $\begin{array}{l}\text { Hetero- } \\
\text { geneity } \\
(p)\end{array}$ & AUC \\
\hline $\begin{array}{l}\text { CEA + } \\
\text { CA } 125\end{array}$ & 3 & $\begin{array}{l}107 / \\
167\end{array}$ & $\begin{array}{l}0.65 \\
(0.56-0.74)\end{array}$ & $\begin{array}{l}6.62 \\
(0.04)\end{array}$ & $\begin{array}{l}0.98 \\
(0.94-0.99)\end{array}$ & $\begin{array}{l}9.40 \\
(0.01)\end{array}$ & $\begin{array}{l}25.20 \\
(4.07-156.07)\end{array}$ & $\begin{array}{l}4.53 \\
(0.10)\end{array}$ & $\begin{array}{l}0.35 \\
(0.21-0.57)\end{array}$ & $\begin{array}{l}6.07 \\
(<0.05)\end{array}$ & $\begin{array}{l}66.08 \\
(16.17-269.40)\end{array}$ & $\begin{array}{l}2.58 \\
(0.28)\end{array}$ & 0.86 \\
\hline $\begin{array}{l}\mathrm{CEA}+ \\
\mathrm{CA} \mathrm{15-3}\end{array}$ & 4 & $\begin{array}{l}272 / \\
443\end{array}$ & $\begin{array}{l}0.64 \\
(0.58-0.70)\end{array}$ & $\begin{array}{l}3.51 \\
(0.32)\end{array}$ & $\begin{array}{l}0.98 \\
(0.97-0.99)\end{array}$ & $\begin{array}{l}6.58 \\
(0.09)\end{array}$ & $\begin{array}{l}28.81 \\
(13.56-61.19)\end{array}$ & $\begin{array}{l}3.48 \\
(0.32)\end{array}$ & $\begin{array}{l}0.37 \\
(0.32-0.43)\end{array}$ & $\begin{array}{l}2.92 \\
(0.41)\end{array}$ & $\begin{array}{l}92.38 \\
(43.09-198.06)\end{array}$ & $\begin{array}{l}1.94 \\
(0.59)\end{array}$ & 0.88 \\
\hline $\begin{array}{l}\mathrm{CEA}+ \\
\mathrm{CA} \mathrm{19-9}\end{array}$ & 4 & $\begin{array}{l}264 / \\
355\end{array}$ & $\begin{array}{l}0.58 \\
(0.51-0.64)\end{array}$ & $\begin{array}{l}34.35 \\
(<0.001)\end{array}$ & $\begin{array}{l}0.98 \\
(0.96-0.99)\end{array}$ & $\begin{array}{l}12.95 \\
(0.01)\end{array}$ & $\begin{array}{l}32.29 \\
(7.48-139.34)\end{array}$ & $\begin{array}{l}6.89 \\
(0.08)\end{array}$ & $\begin{array}{l}0.43 \\
(0.30-0.61)\end{array}$ & $\begin{array}{l}18.26 \\
(<0.001)\end{array}$ & $\begin{array}{l}112.22 \\
(17.88-704.41)\end{array}$ & $\begin{array}{l}9.22 \\
(0.03)\end{array}$ & 0.96 \\
\hline $\begin{array}{l}\text { CEA + } \\
\text { CYFRA 21-1 }\end{array}$ & 7 & $\begin{array}{l}325 / \\
415\end{array}$ & $\begin{array}{l}0.82 \\
(0.77-0.86)\end{array}$ & $\begin{array}{l}36.06 \\
(<0.001)\end{array}$ & $\begin{array}{l}0.92 \\
(0.89-0.95)\end{array}$ & $\begin{array}{l}29.90 \\
(<0.001)\end{array}$ & $\begin{array}{l}8.66 \\
(4.77-15.73)\end{array}$ & $\begin{array}{l}16.85 \\
(0.01)\end{array}$ & $\begin{array}{l}0.18 \\
(0.09-0.34)\end{array}$ & $\begin{array}{l}39.81 \\
(<0.001)\end{array}$ & $\begin{array}{l}68.73 \\
(27.76-170.17)\end{array}$ & $\begin{array}{l}13.72 \\
0.03)\end{array}$ & 0.95 \\
\hline $\begin{array}{l}\text { CA 15-3+ } \\
\text { CYFRA 21-1 }\end{array}$ & 4 & $\begin{array}{l}139 / \\
159\end{array}$ & $\begin{array}{l}0.885 \\
(0.820-0.933)\end{array}$ & $\begin{array}{l}35.71 \\
(<0.001)\end{array}$ & $\begin{array}{l}0.94 \\
(0.89-0.97)\end{array}$ & $\begin{array}{l}15.14 \\
(0.002)\end{array}$ & $\begin{array}{l}8.83 \\
(4.09-19.09)\end{array}$ & $\begin{array}{l}5.03 \\
(0.17)\end{array}$ & $\begin{array}{l}0.06 \\
(0.01-0.59)\end{array}$ & $\begin{array}{l}21.06 \\
(<0.001)\end{array}$ & $\begin{array}{l}300.99 \\
(74.38-1,217.90)\end{array}$ & $\begin{array}{l}0.37 \\
(0.95)\end{array}$ & 0.98 \\
\hline
\end{tabular}

CEA, carcinoembryonic antigen; CA, carbohydrate antigen; N1, number of studies; N2, number of participants (malignant pleural effusion/benign pleural effusion); CI, confidence interval; PLR, positive likelihood ratio; NLR, negative likelihood ratio; DOR, diagnostic odds ratio; AUC, area under the curve. ${ }^{\mathrm{a}} \mathrm{Q}$ value.

Table 2. Weighted meta-regression of the effect of methodological quality on diagnostic precision of combinations of pleural tumor markers

\begin{tabular}{|c|c|c|c|c|}
\hline & Number of studies & Coefficient & RDOR (95\% CI) & $p$ value \\
\hline CEA + CA 125 studies & 3 & & & \\
\hline STARD score $\geq 13$ & 2 & -1.676 & $0.19(0.00-121,179,668.00)$ & 0.485 \\
\hline QUADAS score $\geq 10$ & 2 & -1.676 & $0.19(0.00-121,179,668.00)$ & 0.485 \\
\hline CEA + CA 15-3 studies & 4 & & & \\
\hline STARD score $\geq 13$ & 4 & & & \\
\hline QUADAS score $\geq 10$ & 3 & -0.961 & $0.38(0.00-239.47)$ & 0.587 \\
\hline CEA + CA 19-9 studies & 4 & & & \\
\hline STARD score $\geq 13$ & 4 & & & \\
\hline QUADAS score $\geq 10$ & 3 & -0.137 & $0.87(0.00-32,524.92)$ & 0.960 \\
\hline CEA + CYFRA 21-1 studies & 7 & & & \\
\hline STARD score $\geq 13$ & 5 & 1.085 & $2.21(0.14-35.99)$ & 0.497 \\
\hline QUADAS score $\geq 10$ & 6 & 0.945 & $2.57(0.08-84.52)$ & 0.518 \\
\hline CA 15-3 + CYFRA 21-1 studies & 4 & & & \\
\hline STARD score $\geq 13$ & 4 & & & \\
\hline QUADAS score $\geq 10$ & 3 & -0.675 & $0.51(0.00-880.11)$ & 0.735 \\
\hline
\end{tabular}

RDOR, relative diagnostic odds ratio; CI, confidence interval; CEA, carcinoembryonic antigen; CA, carbohydrate antigen; STARD, Standards for Reporting of Diagnostic Accuracy; QUADAS, Quality Assessment for Studies of Diagnostic Accuracy.

all sensitivities and specificities of the included studies were indicated directly in each publication, our metaanalysis showed that the AUCs of CEA + CA 125, CEA + CA 15-3, CEA + CA 19-9, CEA + CYFRA 21-1, and CA 15-3 + CYFRA 21-1 were 0.86, 0.88, 0.96, 0.95, and 0.98, respectively (see online suppl. Appendix Fig. 1).
Multiple Regression Analysis and Publication Bias

Both QUADAS and STARD scores were used in the meta-regression analysis to assess the effect of study quality on relative DOR for the 5 types of combined tumor markers in diagnosing MPE (Table 2). Studies of higher quality (STARD score $\geq 13$ or QUADAS score $\geq 10$ ) did 
not show an advantage over those of lower quality (STARD score $<13$ or QUADAS score $<10$ ) (all $p$ values $>0.05$ ). Differences between studies with and those without a blinded design or with regard to whether they were consecutive/random, cross-sectional, or prospective did not reach statistical significance (data not shown).

The result of the evaluation of publication bias was not significant for CEA + CA $125(p=0.385)$, CEA + CA 15-3 $(p=0.320)$, CEA + CA 19-9 $(p=0.239)$, or CEA + CYFRA 21-1 $(p=0.122)$, but it was significant for CA 15-3 + CYFRA 21-1 $(p=0.006)$ in the diagnosis of MPE by Egger's test. Funnel plots are shown in online supplementary Appendix Figure 2.

\section{Discussion}

Analysis of tumor markers is considered a minimally invasive method for differentiating between malignant and nonmalignant pleural effusions. However, the search for highly accurate tumor markers in pleural fluid that reliably confirm MPE has been fruitless so far. The diagnostic accuracy of a single tumor marker was shown to be lower than expected, and invasive tests are not always effective [33]. The aim of this study was to evaluate the diagnostic performance of different combinations of pleural tumor markers.

The overall specificity of all the tumor marker groups was more than 0.90, except for CA 19-9 + CYFRA 21-1 and CEA + CA 19-9 + CYFRA 21-1. However, the sensitivity of all combinations was low and variable, ranging from 0.58 to 0.95 . The trade-off between sensitivity and specificity has significant clinical implications for the use of combined tumor markers in differentiating MPE from non-MPE. The sensitivity is higher than that of single markers (CEA, 0.549; CA 15-3, 0.507; CA 19-9, 0.376; CA 125, 0.575; CYFRA 21-1, 0.625) [7], but not by a statistically significant margin. The low sensitivity of any of the tumor marker combinations restricts the clinical applicability of these tests dramatically. These results suggest that combined pleural markers may play more important roles than single markers in diagnosing MPE, but patients with negative results should not be prevented from further, more invasive testing, such as by cytology sampling or pleural biopsies. The specificity of CEA + CA 15-3 and CEA + CA 19-9 was 0.98 and 0.98 , respectively, which means that patients with positive results for those 2 combinations should be strongly suspected of MPE. CEA + CA 15-3 + CA 19-9 showed a specificity of 0.97 and a sensitivity of 0.71 . However, be-

Combinations of Tumor Markers for MPE cause only 1 reference was included in this group, further studies are needed.

The SROC curve presents a summary of test performance and shows the trade-off between sensitivity and specificity. The AUC is currently considered to be the standard method for assessing the accuracy of distribution models. The AUCs of CEA + CA 125, CEA + CA 153, CEA + CA 19-9, CEA + CYFRA 21-1, and CA 15-3 + CYFRA $21-1$ were $0.86,0.88,0.96,0.95$, and 0.98 , respectively, which was much higher than the values of the individual tumor markers (CEA, 0.81; CA 15-3, 0.78; CA 19-9, 0.91; CA 125, 0.79; CYFRA 21-1, 0.84) [7]. The maximum joint sensitivity and specificity - the point of intersection of the SROC curves [34] - of these combinations was $0.79,0.81,0.90,0.89$, and 0.95 , respectively. Together, these data suggest that the overall accuracy of combined tumor markers in diagnosing MPE is higher than their individual accuracy.

DOR is a measure of the effectiveness of a diagnostic test [35]. The value of a DOR ranges from 0 to infinity, with a higher value indicating a better discriminatory test performance (higher accuracy). In this meta-analysis, the DOR values for CEA + CA 125, CEA + CA 15-3, CEA + CA 19-9, CEA + CYFRA 21-1, and CA 15-3 + CYFRA $21-1$ were $66.08,92.38,112.22,68.73$, and 300.99 , respectively, which was much higher than the values for the tumor markers individually (CEA, 30.8; CA 15-3, 59.5; CA 19-9, 29.5; CA 125, 17.4; CYFRA 21-1, 22.8) [7], also indicating that measurement of combined tumor markers would be more helpful than testing tumor markers individually.

To improve the clinical applicability of the results, likelihood ratios were generated for each combination of pleural tumor markers. A likelihood ratio $>10$ or $<0.1$ indicates a 10-fold shift in the probability of the presence of a condition before versus after the diagnostic test. Our data showed that the overall PLR values of CEA + CA 125, CEA + CA 15-3, CEA + CA 19-9, CEA + CYFRA 21-1, and CA 15-3 + CYFRA 21-1 were 25.20, 28.81, 32.29, 8.66, and 8.83 , respectively. These figures suggest that patients with MPE have an approximately 25-, 29-, 32-, 9-, and 9-fold higher chance of testing positive for CEA + CA 125, CEA + CA 15-3, CEA + CA 19-9, CEA + CYFRA 21 1, and CA 15-3 + CYFRA 21-1, respectively, than do patients without MPE. On the other hand, the mean NLR values of CEA + CA 125, CEA + CA 15-3, CEA + CA 199, CEA + CYFRA 21-1, and CA 15-3 + CYFRA 21-1 were $0.35,0.37,0.43,0.18$, and 0.06 , respectively. Therefore, if the assay results of CEA + CA 125, CEA + CA 15-3, CEA + CA 19-9, CEA + CYFRA 21-1, and CA 15-3 + CYFRA 
21-1 are negative, the probability of patients having MPE is approximately $35,37,43,18$, and $6 \%$, respectively. This observation indicates that measurement of these combinations of tumor markers could be helpful in the diagnosis of MPE.

In our meta-regression analysis, STARD and QUADAS scores were used to assess the effect of study quality for all types of combinations of tumor markers. The overall study quality assessed by the QUADAS-2 tool showed a low risk of bias, except for studies that lacked information on their case-control design. We did not find that studies of higher quality had a better test performance than those of lower quality. Differences between studies with and those without a blinded design, or with regard to whether they had a cross-sectional, consecutive/random, or prospective design, did not reach statistical significance.

Our meta-analysis has some limitations. First, for many of the combined tumor marker analyses, the number of cases was small. Therefore, the results of these trials in a pooled analysis are not robust. More studies are needed for future analyses. Second, a publication bias may exist because of the exclusion of conference abstracts, letters to journal editors, and non-English studies. These exclusions may have inflated the estimated diagnostic value due to preferential publication of studies with favorable results. Third, we did not address issues such as reliability, costeffectiveness, the incremental benefit of adding tumor marker assays to other tests, or the net effect of tumor marker assays on clinical care and patient outcomes. Additionally, because the original publications lacked the required parameters, we could not analyze the effect of factors such as patient spectrum, laboratory infrastructure, expertise with tumor marker assay technology, or setting on the accuracy of the tumor marker measurements.
The combinations of tumor markers in our study are highly specific; however, low sensitivity limits their clinical value. Based on the findings from our meta-analysis, in patients with positive CEA + CA 15-3 or CEA + CA 19-9, there is high suspicion of MPE. However, we cannot recommend using any combination of tumor markers for the diagnosis of MPE. Cytology or histology will always be necessary to guide clinical management, even if the tumor markers are positive.

In conclusion, current evidence suggests that the diagnostic accuracy of combinations of 2 or more tumor markers is higher than that of any single tumor marker. The combinations of 2 or more tumor markers are highly specific but insufficiently sensitive for diagnosing MPE. In patients with positive results for CEA + CA 15-3 or CEA + CA 19-9, there is high suspicion of MPE, but management decisions should depend on positive cytological or biopsy results from the pleura.

\section{Acknowledgments}

H.-Z.S. designed the study, analyzed the results, and revised the manuscript; Y.-L.L. and Y.Y. worked equally in study abstraction, data analysis, and main text composition; Y.-L.L. guided the data analysis methods, preparation of all figures, and design of the data abstraction form and served as a second reviewer in extracting the data. All authors reviewed the manuscript.

\section{Financial Disclosure and Conflicts of Interest}

This work was supported in part by grants from the National Natural Science Foundation of China (No. 91442109, No. 31470883 , and No. 81270149). None of the authors have a financial relationship with a commercial entity that has an interest in the subject of this manuscript.

\section{References}

1 American Thoracic Society: Management of malignant pleural effusions. Am J Respir Crit Care Med 2000;162:1987-2001.

2 Bennett R, Maskell N: Management of malignant pleural effusions. Curr Opin Pulm Med 2005;11:296-300.

3 Loddenkemper R: Thoracoscopy - state of the art. Eur Respir J 1998;11:213-221.

4 Alemán C, Sanchez L, Alegre J, Ruiz E, Vázquez A, Soriano T, Sarrapio J, Teixidor J, Andreu J, Felip E, Armadans L, Fernández De Sevilla T: Differentiating between malignant and idiopathic pleural effusions: the value of diagnostic procedures. QJM 2007;100:351-359.
5 Fenton KN, Richardson JD: Diagnosis and management of malignant pleural effusions. Am J Surg 1995;170:69-74.

6 Liang QL, Shi HZ, Qin XJ, Liang XD, Jiang J, Yang HB: Diagnostic accuracy of tumour markers for malignant pleural effusion: a meta-analysis. Thorax 2008;63:35-41.

7 Nguyen AH, Miller EJ, Wichman CS, Berim IG, Agrawal DK: Diagnostic value of tumor antigens in malignant pleural effusion: a meta-analysis. Transl Res 2015;166:432-439.

8 Bossuyt PM, Reitsma JB, Bruns DE, Gatsonis CA, Glasziou PP, Irwig LM, Moher D, Rennie D, de Vet HC, Lijmer JG: The STARD state- ment for reporting studies of diagnostic accuracy: explanation and elaboration. Ann Intern Med 2003;138:W1-W12.

9 Whiting PF, Rutjes AW, Westwood ME, Mallett S, Deeks JJ, Reitsma JB, Leeflang MM, Sterne JA, Bossuyt PM: QUADAS-2: a revised tool for the quality assessment of diagnostic accuracy studies. Ann Intern Med 2011;155: 529-536.

10 Devillé WL, Buntinx F, Bouter LM, Montori VM, de Vet HC, van der Windt DA, Bezemer PD: Conducting systematic reviews of diagnostic studies: didactic guidelines. BMC Med Res Methodol 2002;2:9. 
11 Vamvakas EC: Meta-analyses of studies of the diagnostic accuracy of laboratory tests: a review of the concepts and methods. Arch Pathol Lab Med 1998;122:675-686.

12 Irwig L, Macaskill P, Glasziou P, Fahey M: Meta-analytic methods for diagnostic test accuracy. J Clin Epidemiol 1995;48:119-130; discussion 131-132.

13 Li CS, Cheng BC, Ge W, Gao JF: Clinical value of CYFRA21-1, NSE, CA15-3, CA19-9 and CA125 assay in the elderly patients with pleural effusions. Int J Clin Pract 2007;61:444448 .

14 Wagner IC, Guimarães MJ, da Silva LK, de Melo FM, Muniz MT: Evaluation of serum and pleural levels of the tumor markers CEA, CYFRA21-1 and CA 15-3 in patients with pleural effusion (in English, Portuguese). J Bras Pneumol 2007;33:185-191.

15 Gaspar MJ, De Miguel J, García Díaz JD, Díez M: Clinical utility of a combination of tumour markers in the diagnosis of malignant pleural effusions. Anticancer Res 2008;28:29472952.

16 Hackbarth JS, Murata K, Reilly WM, Algeciras-Schimnich A: Performance of CEA and CA19-9 in identifying pleural effusions caused by specific malignancies. Clin Biochem 2010;43:1051-1055.

17 Huang WW, Tsao SM, Lai CL, Su CC, Tseng CE: Diagnostic value of Her-2/neu, Cyfra 211 , and carcinoembryonic antigen levels in malignant pleural effusions of lung adenocarcinoma. Pathology 2010;42:224-228.

18 Farag DH, El Hadidi E, El Maraghy MO, Hussein MM: Pleural CYFRA 21-1 and CA 15-3 in differentiation of malignant from benign pleural effusions. Life Sci J 2012;9:499-505.

19 Filiberti R, Parodi S, Libener R, Ivaldi GP, Canessa PA, Ugolini D, Bobbio B, Marroni P: Diagnostic value of mesothelin in pleural fluids: comparison with CYFRA 21-1 and CEA. Med Oncol 2013;30:543.
20 Hsieh TC, Huang WW, Lai CL, Tsao SM, Su CC: Diagnostic value of tumor markers in lung adenocarcinoma-associated cytologically negative pleural effusions. Cancer Cytopathol 2013;121:483-488.

21 Son SM, Han HS, An JY, Choe KH, Lee KM, Lee KH, Kim SS, Lee YM, Lee HC, Song HG, Lee OJ: Diagnostic performance of CD66c in lung adenocarcinoma-associated malignant pleural effusion: comparison with CEA, CA 19-9, and CYFRA 21-1. Pathology 2015;47: 123-129.

22 Niwa Y, Shimokata K: Diagnostic significance of cancer antigen 125, pancreatic oncofetal antigen, and carcinoembryonic antigen in malignant and tuberculous pleural effusions. Jpn J Clin Oncol 1986;16:3-8.

23 Ferrer J, Villarino MA, Encabo G, Felip E, Bermejo B, Vilà S, Orriols R: Diagnostic utility of CYFRA 21-1, carcinoembryonic antigen, CA 125, neuron specific enolase, and squamous cell antigen level determinations in the serum and pleural fluid of patients with pleural effusions. Cancer 1999;86:1488-1495.

24 Sthaneshwar P, Yap SF, Jayaram G: The diagnostic usefulness of tumour markers CEA and CA-125 in pleural effusion. Malays J Pathol 2002;24:53-58.

25 Romero S, Fernández C, Arriero JM, Espasa A, Candela A, Martín C, Sánchez-Payá J: CEA, CA 15-3 and CYFRA 21-1 in serum and pleural fluid of patients with pleural effusions. Eur Respir J 1996;9:17-23.

26 Villena V, López-Encuentra A, Echave-Sustaeta J, Martín-Escribano P, Ortuño-de-Solo B, Estenoz-Alfaro J: Diagnostic value of CA 72-4, carcinoembryonic antigen, CA 15-3, and CA 19-9 assay in pleural fluid. A study of 207 patients. Cancer 1996;78:736-740.
27 Villena V, López-Encuentra A, Echave-Sustaeta J, Martín-Escribano P, Ortuño-de-Solo B, Estenoz-Alfaro J: Diagnostic value of CA 549 in pleural fluid. Comparison with CEA, CA 15.3 and CA 72.4. Lung Cancer 2003;40: 289-294.

28 Tozkoparan E, Özkan M, Çiftçi F, Lyl L: The role of serum and pleural fluid levels of Cyfra-21-1, carcinoembryonic antigen and neuron specific enolase in clinical evaluation of pleural effusions for malignancy. Arch Lung 2004;5:194-199.

29 Lee JH, Chang JH: Diagnostic utility of serum and pleural fluid carcinoembryonic antigen, neuron-specific enolase, and cytokeratin 19 fragments in patients with effusions from primary lung cancer. Chest 2005; 128:22982303.

30 Kuralay F, Tokgöz Z, Cömlekci A: Diagnostic usefulness of tumour marker levels in pleural effusions of malignant and benign origin. Clin Chim Acta 2000;300:43-55.

31 Lai RS, Chen CC, Lee PC, Lu JY: Evaluation of cytokeratin 19 fragment (CYFRA 21-1) as a tumor marker in malignant pleural effusion. Jpn J Clin Oncol 1999;29:421-424.

32 Alataş F, Alataş O, Metintaș M, Colak O, Harmanci E, Demir S: Diagnostic value of CEA, CA 15-3, CA 19-9, CYFRA 21-1, NSE and TSA assay in pleural effusions. Lung Cancer 2001;31:9-16.

33 Porcel JM, Light RW: Diagnostic approach to pleural effusion in adults. Am Fam Physician 2006;73:1211-1220.

34 Moses LE, Shapiro D, Littenberg B: Combining independent studies of a diagnostic test into a summary ROC curve: data-analytic approaches and some additional considerations. Stat Med 1993;12:1293-1316.

35 Glas AS, Lijmer JG, Prins MH, Bonsel GJ, Bossuyt PM: The diagnostic odds ratio: a single indicator of test performance. J Clin Epidemiol 2003;56:1129-1135.
Combinations of Tumor Markers for MPE
Respiration 2017;94:62-69 DOI: $10.1159 / 000468545$ 